\title{
Tecnologias utilizadas em cuidados críticos no combate a Covid-19: Revisão de
}

\section{escopo}

\author{
Technologies used in critical care in combating Covid-19: Scope review \\ Tecnologías utilizadas em cuidados críticos para combatir el Covid-19: Revisión del alcance
}

Recebido: 23/02/2021 | Revisado: 03/03/2021 | Aceito: 03/03/2021 | Publicado: 11/03/2021

\author{
Victoria Dorneles Nery \\ ORCID: https://orcid.org/0000-0001-7442-0364 \\ Universidade Federal de São Paulo, Brasil \\ E-mail: victoriadnery@gmail.com \\ Regimar Carla Machado \\ ORCID: https://orcid.org/0000-0001-6126-7663 \\ Universidade Federal de São Paulo, Brasil \\ E-mail: regimar.carla@unifesp.br \\ Camila Bomfim Von Jakitsh \\ ORCID: https://orcid.org/0000-0002-2530-7661 \\ Universidade Federal de São Paulo, Brasil \\ E-mail: cacavonj@hotmail.com \\ Michelle Mayumi Yoshimura de Campos \\ ORCID: https://orcid.org/0000-0002-6256-048X \\ Universidade Federal de São Paulo, Brasil \\ E-mail: michellemyoshimura@gmail.com \\ João Carlos Marchiori de Claudio \\ ORCID: https://orcid.org/0000-0003-0571-1058 \\ Universidade Federal de São Paulo, Brasil \\ E-mail: joaocarlosmdec@gmail.com \\ Rosemeire Simone Dellacrode Giovanazzi \\ ORCID: https://orcid.org/0000-0001-7976-4422 \\ Universidade do Oeste Paulista, Brasil \\ E-mail: sgiovanazzi@gmail.com \\ Suelen Alves Creste Martins da Costa \\ ORCID: https://orcid.org/0000-0001-9848-5672 \\ Universidade do Oeste Paulista, Brasil \\ E-mail: suelencreste@gmail.com
}

\begin{abstract}
Resumo
Objetivo: buscar na literatura científica as tecnologias a serem utilizadas por profissionais de enfermagem no atendimento de alta complexidade para o combate à doença ocasionada pelo novo Coronavírus (SARS-Cov2). Método: revisão no formato de scoping review baseado nos procedimentos recomendados pelo Instituto Joanna Briggs. Realizou-se mapeamento nas bases de literatura branca e cinzenta, em um recorte atemporal e sem limitação de idioma. O delineamento de seleção dos estudos seguiu duas triagens com a finalidade de diminuição de viés. Os estudos incluídos foram apresentados como sínteses narrativas e tabelas. Resultados: foram mapeadas 680 publicações, das quais quatro estudos foram selecionados por contemplarem todos os critérios de inclusão. Os achados mais relevantes envolvem a utilização de luz ultravioleta, radiação, oxigenação por membrana extracorpórea e ecocardiogramas portáteis. Conclusão: observou-se poucas, porém promissoras tecnologias estudadas para utilização na assistência de alta complexidade à paciente com a COVID-19, mostrando-se um campo carente e favorável à pesquisa.
\end{abstract}

Palavras-chave: Infecções por coronavírus; Tecnologia biomédica; Cuidados críticos; Unidades de terapia intensiva; Revisão.

\begin{abstract}
Objective: to search the scientific literature for technologies to be used by nursing professionals in high complexity care to combat the disease caused by the new Coronavirus (SARS-Cov2). Method: review in the scoping review format based on the procedures recommended by the Joanna Briggs Institute. Mapping was carried out on the bases of white and gray literature, in a timeless cut and without language limitation. The study selection design followed two screenings in order to reduce bias. The included studies were presented as narrative summaries and tables. Results: 680 publications were mapped, of which four studies were selected because they met all the inclusion criteria. The most relevant findings involve the use of ultraviolet light, radiation, extracorporeal membrane oxygenation and
\end{abstract}


portable echocardiograms. Conclusion: there were few, but promising, technologies studied for use in highly complex assistance to patients with COVID-19, showing a needy and favorable field for research.

Keywords: Coronavirus infections; Biomedical technology; Critical care; Intensive care units; Review.

\begin{abstract}
Resumen
Objetivo: buscar en la literatura científica tecnologías a ser utilizadas por los profesionales de enfermería en cuidados de alta complejidad para combatir la enfermedad causada por el nuevo coronavirus (SARS-Cov2). Método: revisión en el formato de revisión del alcance con base en los procedimientos recomendados por el Instituto Joanna Briggs. El mapeo se realizó sobre la base de la literatura blanca y gris, en un corte atemporal y sin limitación de idioma. El diseño de selección del estudio siguió dos evaluaciones para reducir el sesgo. Los estudios incluidos se presentaron como resúmenes narrativos y tablas. Resultados: se mapearon 680 publicaciones, de las cuales se seleccionaron cuatro estudios por cumplir con todos los criterios de inclusión. Los hallazgos más relevantes involucran el uso de luz ultravioleta, radiación, oxigenación por membrana extracorpórea y ecocardiogramas portátiles. Conclusión: existían pocas, pero prometedoras, tecnologías estudiadas para su uso en la asistencia de alta complejidad a pacientes con COVID-19, mostrando un campo de investigación necesitado y favorable.
\end{abstract}

Palabras clave: Infecciones por coronavirus; Tecnología biomédica; Cuidados críticos; Unidades de cuidados intensivos; Revisión.

\title{
1. Introdução
}

Nos últimos vinte anos, três variedades de coronavírus causaram crises de saúde pública no cenário mundial. Inicialmente foi descrito o SARS-CoV na China em 2003, com taxa de mortalidade de nove e meio por cento (9,5\%), seguindo-se do MERS-CoV no Oriente Médio em 2011, com mortalidade de 35\% e do SARS-CoV-2 no final 2019, com o primeiro caso notificado na cidade de Wuhan, China e mortalidade estimada de dois por cento (2\%). Apesar da menor mortalidade decorrente do SARS-CoV-2, a sua alta transmissibilidade (1:2-3) se comparado à de seus antecessores (1:1) é o que gera impacto desfavorável à saúde pública mundial. Ademais, um agravante à doença foi o local de origem da infecção por SARS-CoV-2, que por tratar-se de em uma metrópole habitada por mais de 11 milhões de pessoas favoreceu a disseminação do vírus (Guarner, 2020).

A alta transmissibilidade do SARS-CoV-2 ocasionou um aumento no número de infectados, favorecendo a propagação da doença para outros continentes em um curto período de tempo. Consequentemente diante da magnitude do problema houve a necessidade de uma resposta global coordenada de saúde, sendo declarado pela Organização Mundial da Saúde (OMS), tratar-se de uma emergência de saúde pública de importância internacional, em 30 de janeiro de 2020(OPAS, 2020).

Atualmente contabilizam-se 91 milhões de casos confirmados e mais de um milhão de mortes por "coronavírus disease 2019" (COVID-19), conforme relatório de 18 de janeiro de 2021 da OMS (WHOa, 2020). Em 80\% dos casos, os sintomas são leves: febre, tosse seca, falta de ar e fadiga, porém, o agravamento do quadro clínico pode ocasionar à síndrome respiratória aguda grave, decorrente de uma pneumonia severa. A disfunção pulmonar compromete a hematose, levando à necessidade da assistência hospitalar de alta complexidade para melhora clínica, sendo as Unidades de Terapia Intensiva (UTI) os locais apropriados para esse atendimento (BR, 2020; Lima, 2020; Backes et al, 2015).

A UTI é um ambiente dotado de aparato tecnológico e equipe multiprofissional qualificada em cuidados à pacientes graves, instáveis e complexos, que requerem assistência ininterrupta com terapêuticas avançadas, procedimentos invasivos e recursos tecnológicos necessários à estabilidade hemodinâmica (Backes et al, 2015).

$\mathrm{O}$ atendimento ao paciente grave por suas particularidades, demanda a incorporação de tecnologias de pontas ao cuidado, que possibilitam a melhor terapêutica, favorecendo o prognóstico de pacientes críticos diagnosticados com a COVID19 (Cheung et al, 2020).

Ademais, a assistência à saúde, pautada por tecnologia efetivas no combate a COVID-19, busca evitar o colapso do sistema de saúde, por falta de leitos e profissionais qualificados. Corroborando com a diminuição do período de internação de 
pacientes acometidos pela COVID-19, exposição dos profissionais de saúde ao risco de contaminação e impacto da pandemia na saúde pública mundial. Para tal, pesquisas desenvolvidas no cenário global que evidenciem estratégias tecnológicas efetivas no combate a COVID-19, são válidas e devem ser difundidas globalmente. Salienta-se que esta pesquisa vai além de evidenciar tecnologias, mas envolve a análise criteriosa da aplicabilidade destas na busca dos melhores resultados assistenciais a serem replicados em âmbito global.

Sendo assim, o referido artigo tem como objetivo buscar na literatura científica as tecnologias a serem utilizadas pelos profissionais de enfermagem no atendimento de alta complexidade para o combate à doença pelo novo Coronavírus (SARSCov2).

\section{Metodologia}

O estudo se apresenta no formato de Scoping Review, uma revisão sistematizada, exploratória, que tem como objetivo identificar produções científicas relevantes em todas as bases disponíveis sobre o assunto no tema escolhido, no caso tecnologias a serem utilizadas no combate a COVID-19.

Os termos "Preferred Reporting Items for Systematic Reviews and Meta Analyses Extension for Scoping Review" (PRISMA-ScR), foram seguidos com a finalidade de melhorar o relato da revisão e ser o mais claro possível para o leitor (Tricco et al, 2018). Junto a isso, foi protocolado essa revisão no Open Science Foundation com o número 10.17605/OSF.IO/US28K.

Preconizou-se para pesquisa o método mnemônico População, Conceito e Contexto (PCC), que visa a concepção da estratégia de busca mais adequada, construção do título e da pergunta de pesquisa (Peters et al, 2015).

Portanto na estratégia PCC, considerou-se como P - profissionais de saúde e pacientes com diagnóstico suspeito ou confirmado da COVID-19; $\mathrm{C}$ - tecnologias utilizadas na alta complexidade; $\mathrm{C}$ - combate ao novo coronavírus. A busca e a seleção dos estudos foram norteadas de acordo com a pergunta de pesquisa: "Quais tecnologias podem ser utilizadas pelos profissionais de saúde no atendimento de alta complexidade para o combate ao novo coronavírus?".

Para critérios de inclusão, considerou-se estudos disponíveis na íntegra para acesso dos pesquisadores, que apresentaram como amostra profissionais de saúde atuantes na alta complexidade ou pacientes com diagnóstico da COVID-19 submetidos a alta complexidade. Quanto aos critérios de exclusão, não foram selecionados estudos que não respondessem à questão norteadora e/ou não estavam voltados para população de interesse, além de publicações duplicadas.

As estratégias de busca realizaram-se nas diversas bases de dados, como a Pubmed, Web of Science, EMBASE Cochrane Library, Cumulative Index to Nursing and Allied Health Literature (CINAHL), Controlled Trials, Clinical Trials, Science Direct e Open Grey, por artigos publicados na íntegra, sem pré-definição de desenho metodológico, sem limite de idiomas, em um recorte atemporal. Na combinação das palavras chave empregou-se os operadores booleanos AND e OR. O período de pesquisa nas bases de dados foi de 12 a 17 de maio de 2020.

Quanto às palavras chaves utilizadas nesta pesquisa foram; "Technology, Biomedical; Biomedical Technologies; Technology, Health Care; Health Technology; Technology, Health; Health Care Technology; Care, Critical; Intensive Care; Care, Intensive; Surgical Intensive Care; Care, Surgical Intensive; Intensive Care, Surgical; 2019 novel coronavirus disease; COVID19; COVID-19 pandemic; SARS-CoV-2 infection; COVID-19 virus disease; 2019 novel coronavirus infection; 2019nCoV infection; coronavirus disease 2019; coronavirus disease-19; 2019-nCoV disease; COVID-19 virus infection". As estratégias de busca também contaram com filtros disponibilizados nas bases selecionando artigos com foco em humanos e adultos. 
A apresentação do percurso realizado para seleção dos estudos seguiu o fluxograma PRISMA (Tricco et al, 2018), que contempla o total de publicações encontradas nas bases de dados selecionadas, o processo de exclusão de duplicatas, a seleção das publicações de acordo com os critérios de elegibilidade e por fim a amostra de artigos que atenderam ao objetivo desta pesquisa. As revisões de escopo não preveem a exclusão de artigos segundo critérios de qualidade metodológica, mas, para apoiar a síntese e qualidade dos dados selecionados, os artigos selecionados nesta revisão foram submetidos à avaliação de qualidade metodológica por instrumentos do Joanna Briggs Institute (Peters et al, 2015).

$\mathrm{O}$ processo de seleção das publicações envolveu dois revisores independentes $(\mathrm{C}, \mathrm{V})$, aliado a dois instrumentos estruturados elaborados pelos próprios pesquisadores, conforme recomendações do Instituto Joanna Briggs (Peters et al, 2015) (JBI) e aplicado em dois tipos de triagem.

A triagem I realizou-se por meio da análise dos títulos e resumos dos artigos encontrados, assim como avaliação do enquadramento dos mesmos na população, conceitos e contextos padronizados. Na triagem II, foram avaliados artigos por meio da leitura na íntegra e enquadramento dos mesmos na população, conceitos e contextos padronizados. Ambos os testes de triagem foram construídos com espaços complementares para preenchimento das seguintes informações: autores, ano de publicação, país de realização do estudo, país de publicação, revista publicada, área de pesquisa e observações relevantes. Para a triagem I o instrumento foi composto por duas questões, sendo elas "O estudo aborda tecnologias utilizadas pelos profissionais de saúde da alta complexidade no combate a COVID-19?" e "Os sujeitos do estudo são profissionais da saúde atuantes na alta complexidade e/ou pacientes críticos portadores da COVID19?", estas questões deveriam ser respondidas com "sim", "não" ou "não relatado". Em caso de duas respostas afirmativas o estudo foi selecionado para análise na íntegra, no caso de uma resposta negativa o estudo era excluído e no caso de uma resposta contemplar como o item "não relatado" o estudo era analisado na íntegra. Para a triagem II o instrumento contou com a descrição detalhada dos objetivos/hipóteses, método, participantes, intervenções, desfechos, tipo de tecnologia utilizada, resultados e conclusões. A partir da triagem II selecionaram-se estudos que retratavam tecnologias utilizadas e avaliadas para o combate da COVID-19 e excluíram-se estudos que não realizaram análise de tecnologias, que não foram utilizados no combate ao Sars-Cov-2 e que não foram desenhados para a aplicação na alta complexidade. Os instrumentos utilizados para triagem dos artigos foram validados pela metodologia da JBI seguindo o template de fonte de detalhes de evidências sendo adaptados à temática com a finalidade de garantir a qualidade metodológica dos artigos selecionados, reduzindo os possíveis viés do estudo.

Os estudos selecionados para a amostra foram sintetizados para apresentação em formato de quadro descritivo ressaltando os achados mais pertinentes frente às tecnologias utilizadas no combate ao coronavírus - COVID-19. Para a elaboração da pergunta da pesquisa, foi utilizado o método mnemônico população - conceito - contexto (PCC) (Peters, 2020). Dessa forma, obteve-se a pergunta focal: quais as alterações cardiovasculares encontradas em pacientes submetidos à Unidade de Terapia Intensiva com diagnóstico positivo para COVID-19?

\section{Resultados}

A pesquisa identificou 680 estudos de interesse nas bases de dados eletrônicas selecionadas (Figura 1). A plataforma Science Direct foi a que apresentou maior número de estudos na temática com 382 publicações. Em seguida, realizou-se a exclusão de duplicatas, totalizando 675 referências. Destas 624 referências foram descartadas pelo título, resultando em 51 estudos para leitura do resumo. 
Figura 1 - Fluxograma PRISMA (adaptado) do processo de seleção dos artigos.

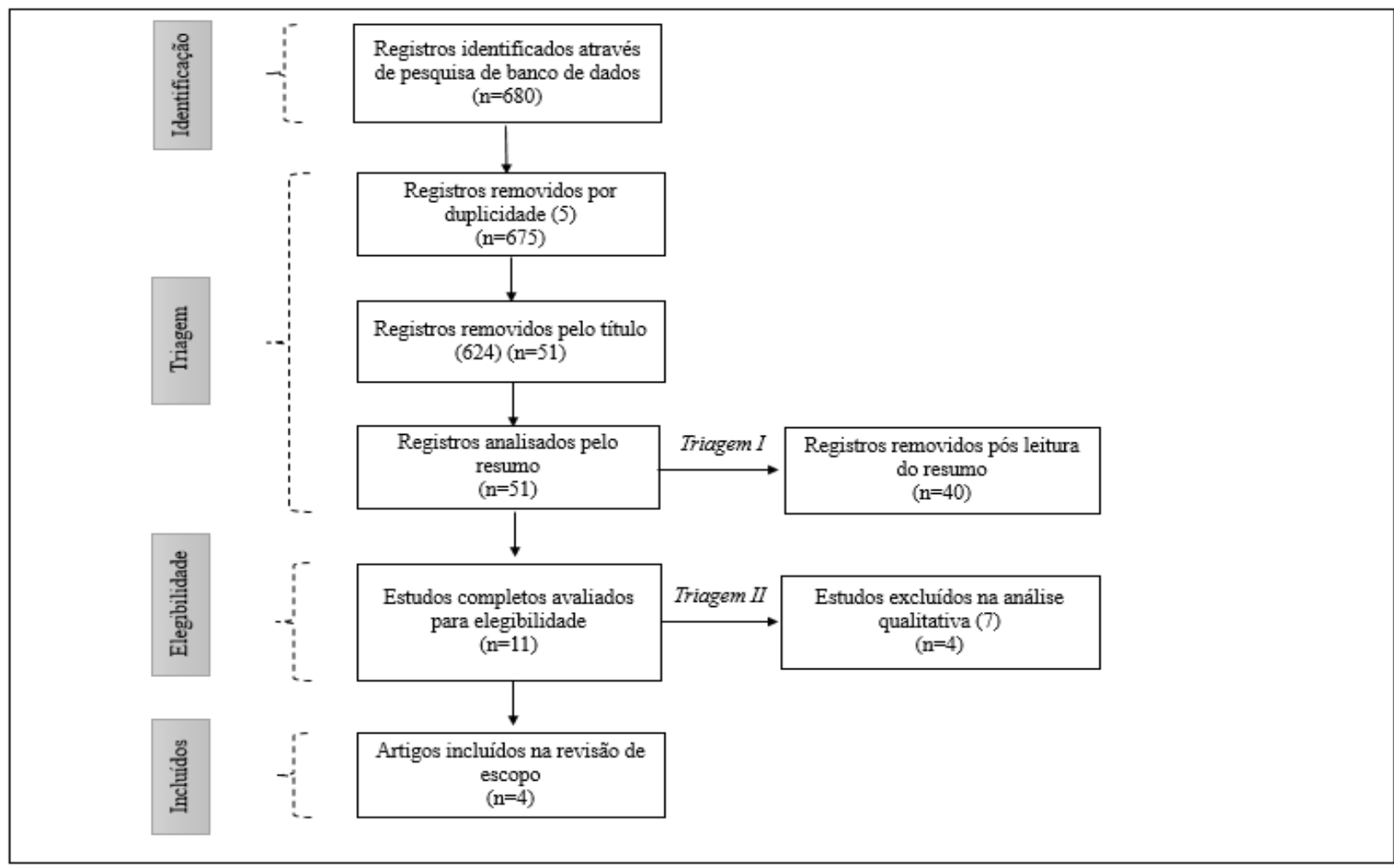

Fonte: Autores.

A partir do processo de triagem I, foram excluídos 40 estudos que responderam negativamente a uma das questões propostas, resultando em 11 estudos elegíveis para serem lidos integralmente e avaliados com o instrumento de triagem II. Após análise qualitativa dos 11 estudos, sete foram excluídos por não preencherem os critérios de inclusão da pesquisa, resultando em quatro artigos incluídos para extração e síntese dos dados.

Os quatro artigos mapeados para pesquisa foram publicados no ano de 2020, dois tiveram como país de estudo os Estados Unidos da América (EUA), um estudo foi realizado no Peru e outro na China. Os tipos de estudos encontrados foram revisão sistemática de literatura, revisão da literatura, artigo original e estudo observacional retrospectivo. A amostra mais representativa foi de 90 pacientes e a menos representativa contou com 12 indivíduos. Os quatro estudos analisaram homens e mulheres com idade superior ou igual a 18 anos e que testaram positivo para COVID-19. A síntese dos dados de interesse encontra-se descritos no Tabela 1.

Tabela 1 - Síntese dos achados dos estudos incluídos nesta revisão de escopo. São Paulo, SP, Brasil, 2020.

\begin{tabular}{ccccccc}
\hline Título ${ }^{(*)}$ & $\begin{array}{c}\text { Ano } \\
\text { País }\end{array}$ & População & $\begin{array}{c}\text { Tipo de } \\
\text { Estudo }\end{array}$ & Intervenções & Desfechos & $\begin{array}{c}\text { Nível de } \\
\text { Evidência* }\end{array}$ \\
\hline $\begin{array}{c}\text { Uso da Luz } \\
\text { Ultravioleta }\end{array}$ & & & A luz & Não foram \\
como & 2020 & \multirow{2}{*}{7 estudos } & sistemática & como substituto & estudos que & II \\
substituto para & Peru & & da literatura & da pressão & forneçam & evidências \\
$\begin{array}{c}\text { pressão negativa } \\
\text { em Unidades de }\end{array}$ & & & Unidada em & científicas do uso & \\
\hline
\end{tabular}




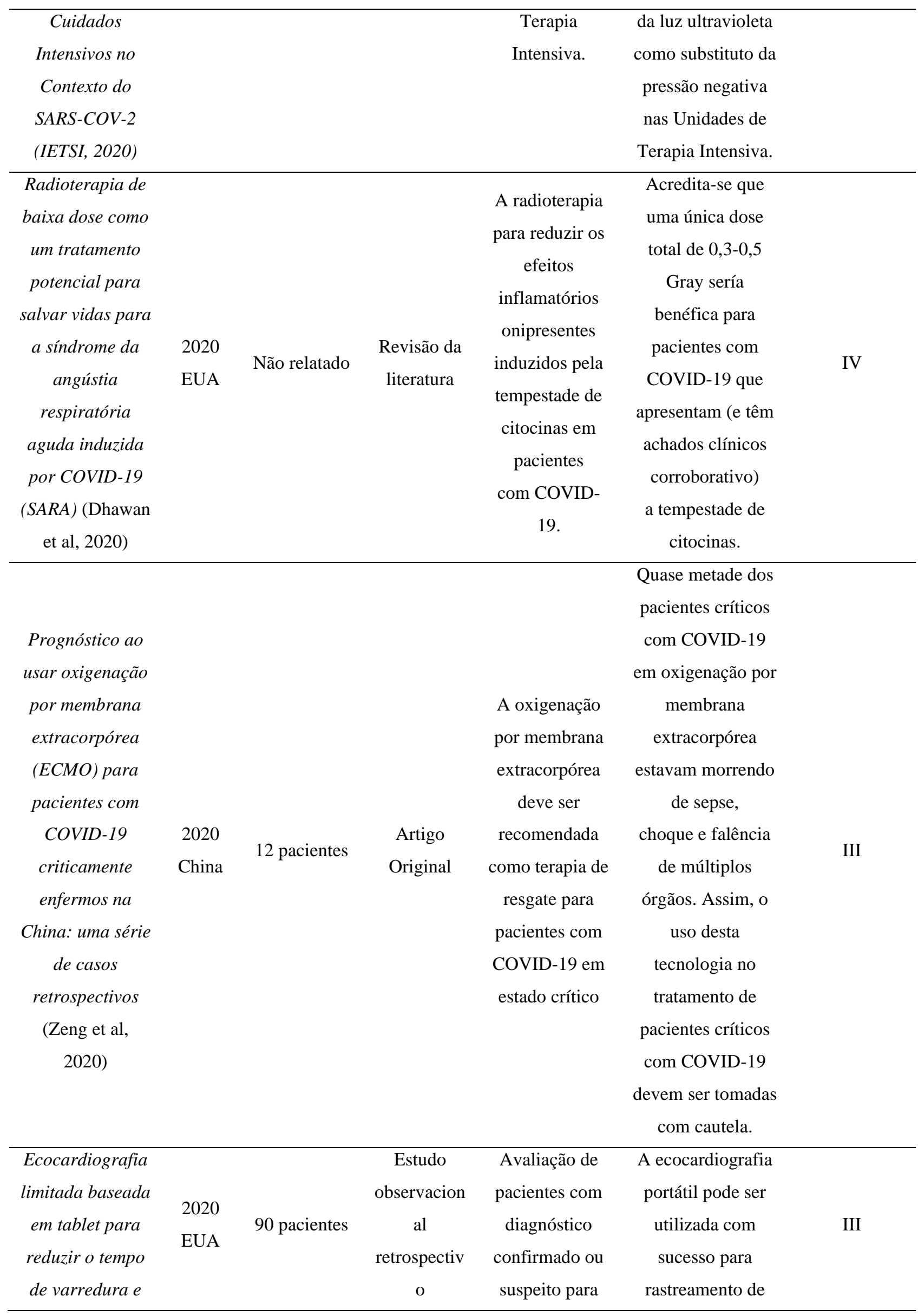


Research, Society and Development, v. 10, n. 3, e18210313243, 2021

(CC BY 4.0) | ISSN 2525-3409 | DOI: http://dx.doi.org/10.33448/rsd-v10i3.13243

\begin{tabular}{|c|c|c|c|}
\hline descontaminação & unicêntrico & COVID-19 & condições \\
\hline por & & utilizando-se & cardíacas \\
\hline ultrassonografia & & imagens & relacionadas à \\
\hline durante a & & ecocardiográfica & SARS-COV-2 \\
\hline pandemia de & & $\mathrm{s}$ realizadas por & \\
\hline COVID-19 & & ultrassom & \\
\hline (McMahon et al, & & portátil. & \\
\hline \multirow[t]{3}{*}{ 2020) } & & Exames & \\
\hline & & ecocardiográfic & \\
\hline & & os abrangentes. & \\
\hline
\end{tabular}

* Nível de Evidência dos estudos em conformidade com o Instituto Joanna Briggs (JBI)

Fonte: Autores.

Quando analisadas as tecnologias para combate a COVID-19 estudadas nos quatro artigos selecionados para esta revisão de escopo, evidenciam-se como promissoras duas delas. O ecocardiograma portátil (McMahon, 2020), que permite adequada avaliação das complicações cardíacas decorrentes da COVID-19, além de favorecer a desinfecção do equipamento e minimizar o risco de contágio por exposição do profissional de saúde e a radioterapia de baixa dosagem (Dhawan et al, 2020), que reduz os efeitos adversos decorrentes da tempestade de citocinas presente na fase aguda da COVID-19, contribuindo com o melhor prognóstico do paciente.

Ademais, os equipamentos portáteis de raios-X são de fácil acesso e encontram-se disponíveis na maioria dos hospitais. Com isso, os pesquisadores ou profissionais de saúde podem produzir radioterapia de baixa dosagem de forma simples, diretamente em pacientes sob ventilação mecânica no ambiente de UTI, com baixo custo e mínimos efeitos colaterais imediatos ou a longo prazo.

Os outros dois estudos analisados (IETSI, 2020; Zeng et al, 2020) apesar de inicialmente viáveis ao combate da COVID-19 não mostraram resultados promissores. Dados da literatura retratam que o componente genético do SARS-CoV-2 é semelhante ao coronavírus MERS e SARS, sendo demonstrado in vitro a inibição do crescimento de ambos os vírus com a aplicação da luz UV (IETSI, 2020). Todavia, a revisão sistemática nesta temática (IETSI, 2020) não encontrou evidências científicas que corroborem com a substituição da pressão negativa na UTI pela aplicação da luz ultravioleta no combate a COVID-19. Da mesma maneira, apesar da ECMO ser recomendada pela OMS para tratamento de pacientes com a COVID-19 que não respondem à terapêutica convencional com ventilação mecânica, estudo retrospectivo realizado na China ${ }^{(12)}$ evidenciou resultados pouco promissores para tecnologia, sendo que pacientes submetidos à ECMO apresentaram os piores desfechos. Salientam -se também que os custos deste tratamento são elevados, chegando a US\$ 73.122 nos EUA (Zeng et al, 2020).

Por fim, os quatro estudos (IETSI, 2020; Dhawan et al, 2020; Zeng et al, 2020; McMahon et al, 2020) consideram serem necessárias novas pesquisas que envolvam a aplicação das tecnologias avaliadas na assistência de paciente com a COVID-19 para corroborar com prática clínica e científica baseada em evidências.

\section{Discussão}

Apesar do cenário mundial de enfrentamento ao novo coronavírus, ainda são poucas as evidências científicas que retratem tecnologias que possam ser utilizadas pelos profissionais de saúde no atendimento de alta complexidade a pacientes com a COVID-19. Observa-se que o escopo da literatura nesta temática contemplou apenas quatro estudos, sendo que dois 
trataram-se de artigos originais (Zeng et al, 2020; McMahon et al, 2020) e dois realizaram revisões da literatura (IETSI, 2020; Dhawan et al, 2020). Quanto à análise da efetividade das tecnologias estudadas, duas mostraram-se promissoras à assistência de pacientes com a COVID-19, sendo estas a radioterapia (Dhawan et al, 2020) e o ecocardiograma portátil (McMahon et al, 2020); enquanto as demais, apesar de inicialmente favoráveis ao combate ao vírus não evidenciam resultados benéficos (IETSI, 2020; Zeng et al, 2020).

No que se refere ao local de realização dos estudos, a maioria das publicações (50\%) foram produzidas nos Estados Unidos da América (EUA), o que se explica pela magnitude da pandemia neste país, que atualmente detém o maior número de casos notificados no cenário mundial (WHOb, 2020).

Ademais, foram as tecnologias evidenciadas por estas publicações que mostraram os resultados mais promissores frente a COVID-19. Os EUA é uma potência tecnológica e científica, sendo detentor de grande número de pesquisas voltadas ao enfrentamento da COVID-19 que culminam com a obtenção dos melhores resultados (Courtemanche et al, 2020; Omary et al, 2020).

Da mesma maneira, a China pela gênese da pandemia no hemisfério asiático, tem despontado nas pesquisas de combate a COVID-19 (WHOb, 2020), todavia a ECMO ao ser avaliada em dois grandes centros de Wuhan, não obteve resultados que embasam sua recomendação em conformidade com a OMS (Zeng et al, 2020). Por se tratar de uma terapêutica com alto custo agregado, a implementação da ECMO é restrita a países que detém maior gama de serviços tecnológicos, em detrimento à países subdesenvolvidos ou em desenvolvimento, em que esta terapêutica se limita a uma minoria da população que tem acesso aos grandes centros de saúde (Loforte et al, 2020).

Por fim, a luz ultravioleta (UV), foi avaliada como uma opção terapêutica para substituição da pressão negativa em UTIs. Neste cenário a luz UV poderia figurar como alternativa para hospitais que não detém este recurso, além de representar uma opção terapêutica de menor custo (Ueki et al, 2006) para o sistema público de saúde e com resultados promissores (IETSI, 2020).

A análise das tecnologias elencadas por esta revisão de escopo, evidencia a utilização da radioterapia (RT) de baixa frequência no tratamento de condições inflamatórias e infecciosas, despontando como uma alternativa viável para reduzir as complicações decorrentes da cascata inflamatória presentes na fase aguda da COVID-19 (IETSI, 2020). Recomenda-se que em pacientes gravemente enfermos à RT de baixa dosagem, seja administrada em dose única de 0,3-0,5 unidade gray (Gy) na região do tórax, podendo induzir a polarização tanto de fenótipos dos macrófagos M1 como M2, o que minimiza o impacto da inflamação sistêmica ocasionada pela COVID-19 (Dhawan et al, 2020). Corroborando com estes achados, retrata-se o uso de radioterapia de baixa frequência para tratamento de pneumonia, em que se observa amenização dos sintomas da doença poucas horas após a aplicação da terapêutica, como consequência da indução de fenótipos anti-inflamatórios (Kirkby \& Mackenzie, 2020).

Salienta-se ainda como benefícios da RT de baixa dosagem (Dhawan et al, 2020) a disponibilidade de equipamento de raio-x portáteis em diversos ambientes hospitalares, mesmo em países com baixo perfil sociodemográfico, como o Brasil (BR, 2009). Todavia, a literatura (Araujo, Sá \& Atty, 2016) evidencia que a implementação de radioterapia efetiva e segura é dependente de equipe profissional qualificada. No cenário brasileiro, há déficit de recursos humanos capacitados nesta terapêutica, com um número menor de profissionais habilitados do que seria necessário, o que impede a realização do tratamento não por falta de recurso tecnológico, mas de qualificação profissional (Brand, Fontana \& Santos, 2011).

Quanto aos efeitos colaterais decorrentes da RT em baixa frequência são mínimos tanto de imediato quanto a longo prazo (Dhawan et al, 2020). Descreve-se como efeitos adversos da RT, reações de pele (radiodermite, eritema), náuseas, mucosite, xerostomia, fadiga, anorexia, diarreia e disfagia (Souza et al, 2017). O conhecimento do enfermeiro acerca dos 
possíveis efeitos colaterais leva ao planejamento de intervenções que minimizem o impacto da RT e favoreçam a assistência efetiva do paciente (Souza et al, 2017).

Outra tecnologia avaliada por esta revisão de escopo foi o ecocardiograma portátil, que se mostrou como uma terapêutica promissora para atendimento de pacientes acometidos pela COVID-19, uma vez que permite a triagem de condições cardíacas relacionadas a essa doença, facilita a desinfecção do equipamento e diminui o tempo de exposição do profissional de saúde (McMahon et al, 2020).

O ecocardiograma portátil ao ser comparado com o ecocardiograma padrão (Telford et al, 2018) representa um método diagnóstico seguro, não invasivo e passível de ser implementado em ambientes remotos e com recursos limitados. Ademais seu uso reduz significantemente o tempo da realização do exame e diminui em até $20 \%$ a necessidade de uma ecocardiografia padrão (Haji et al, 2019). Em detrimento a estes achados, estudo (Ashrafian et al, 2004) realizado em UTI evidenciou que mais de trinta por cento de alterações cardíacas clinicamente relevantes não foram identificados por meio do ecocardiograma portátil, o que se atribui a menor definição da imagem deste equipamento (2D), em escala de cinza e doppler colorido. Além disso, o dispositivo portátil apresenta menor campo de visualização, número limitado de seções de eco e baixa resolução de imagem quando comparado ao exame convencional (Savino \& Ambrosio, 2019).

No que tange os cuidados com o dispositivo portátil, achados da literatura (Zhang et al, 2020) evidenciam que equipamentos alocados em unidades de tratamento de paciente com a COVID-19, devem ser de uso exclusivo da unidade, não podendo ser compartilhados com os demais setores hospitalares. Ressalta-se ainda que após seu uso, a equipe de enfermagem deve realizar a desinfecção do equipamento com peróxido de hidrogênio seguido por exposição à luz ultravioleta (Zhang et al, 2020) que se mostra uma tecnologia efetiva para eliminação do vírus em sua forma vegetativa (Souza, 2014).

Revisão sistemática (IETSI, 2020) sobre a utilização da luz UV como substituta da pressão negativa em UTI, avaliou experimentos in vitro com as diversas formas de luz UV e sua ação na erradicação de vírus, porém não elencou evidências científicas que comprovem que esta tecnologia inativa o SARS-CoV-2, apesar de estudo prévios terem apresentado resultados promissores da ação da luz UV do tipo C na inativação das demais variedade de coronavírus (SARS-CoV e MERS-CoV).

A luz UV do tipo C é definida como luz de onda curta, de ação germicida (Galasso et al, 2019), que não acomete o DNA das células vivas do corpo humano (Wong, Ranganath \& Kasko, 2015). Representa uma tecnologia de baixo custo se comparada à radiação gama ou de alta pressão; não produz odores, é ambientalmente segura; e se mostra com efetiva para descontaminação de superfícies, pois atua na erradicação de microrganismos como vírus, bactérias, fungos, levedos, mofos, algas e protozoários (Souza, 2014; Balbinot Filho \& Borges, 2020). Salienta-se que a luz UVC só pode ser usada por meio de lâmpadas especiais, uma vez que a camada de ozônio da terra filtra quase que completamente essa radiação (Balbinot Filho \& Borges, 2020).

A OMS recentemente incluiu o uso da luz UVC como meio de desinfecção após realização de limpeza manual de ambientes destinados ao atendimento ao paciente com a COVID-19 (WHOc, 2020). Desta forma, entende-se que a luz UVC consegue realizar a eliminação do vírus em sua forma vegetativa dentro do ambiente, mas ainda não se comprova sua eficácia como substituta à pressão negativa.

Como ponto de atenção para a aplicação da luz UVC, ressalta-se que a mesma requer ausência de seres humanos no ambiente, pois este tipo de luz mostra-se prejudicial à pele e aos olhos, podendo causar eritema e queratite (Franco, 2018). Os efeitos mais críticos da luz UVC em humanos tratam-se da carcinogenicidade da pele decorrente de sua genotoxicidade (Franco, 2018). Em contrapartida, estudo (Yamano et al, 2020) recente, com a exposição camundongos à fenótipo altamente foto carcinogênico a longo prazo com a luz UVC de $222 \mathrm{~nm}$, não observou tumores, eritema e edemas na orelha dos animais; sugerindo que as lâmpadas UVC de $222 \mathrm{~nm}$ podem ser seguramente usadas para esterilização de peles humanas sem perspectiva de ocorrência do câncer de pele. 
Somando-se às tecnologias já analisadas a ECMO vem sendo recomendada pela OMS para assistência de pacientes com a COVID-19 que evoluem com síndrome do desconforto respiratório agudo (SDRA) (Jacobs et al, 2020). Entretanto, estudos iniciais nesta temática mostraram-se contrários a recomendação da OMS, evidenciando que pacientes com a COVID que evoluíram com SDRA quanto submetidos à ECMO obtiveram os piores desfechos (Zeng et al, 2020). Em contraponto, na literatura mais recente, desponta a indicação da ECMO no combate a COVID-19 (Loforte et al, 2020; Yamano et al, 2020), uma vez que no cenário atual não há terapêutica adequada para tratamento das complicações decorrentes desta patologia e estabelecer suporte ventilatório efetivo é um desafio à equipe de saúde (Loforte et al, 2020). Neste contexto, a ECMO deve ser considerado quando o tratamento conservador (ventilação mecânica) não se mostrar eficaz (Loforte et al, 2020), sendo os critérios de elegibilidade dos pacientes para esta terapêutica pautados por estudos e diretrizes recentes no assunto, no intuito de mitigar fatores que possam comprometer os resultados (Ramanathan et al, 2020). Aliado à implementação da ECMO, recomenda-se a uso da ventilação mecânica ultra protetora para favorecer o melhor prognóstico do paciente (Ramanathan et al, 2020). Estudos têm apresentado resultados promissores na estabilização e sobrevivência de pacientes críticos com a COVID19 em uso de ECMO (Jacobs et al, 2020).

Diante do exposto, as tecnologias elencadas por esta pesquisa, quando analisadas por achados recentes da literatura, mostram-se promissoras no combate a COVID-19. Não há consenso quanto à efetividade destes recursos, mas se bem indicados seus resultados são promissores. Salienta-se a importância de novas evidências científicas que corroborem com o uso da luz UVC como substituta à pressão negativa em UTI, uma vez que seu papel na erradicação das formas vegetativas do vírus da superfície já está comprovado.

Vale ressaltar as limitações encontradas frente a realização deste estudo, como o período de rastreio dos artigos, uma vez que diariamente surgem novas publicações na temática que não foram incluídas nesta revisão de escopo por encerramento das buscas e o fato de não ter sido realizado o contato com os autores das publicações selecionadas para avaliar a possibilidade de haver outras fontes de dados que possibilitariam obter de informações adicionais.

Ainda, deve-se apresentar que esse estudo apresenta uma grande contribuição para área da saúde pois possibilita a avaliação das tecnologias que despontam no cenário mundial para assistência de pacientes críticos com a COVID-19 embasa a prática clínica da equipe de saúde, além de favorecer subsídios para a construção de diretrizes assistenciais para o enfrentamento da doença.

\section{Conclusão}

Observa-se que o impacto mundial da pandemia pelo novo coronavírus impulsiona as pesquisas na busca por evidências científicas que embasam a assistência prestada à pacientes acometidos pela COVID-19.

Entretanto, apesar dos esforços, ainda são poucas as tecnologias que despontam para utilização na alta complexidade, porém estas mostraram-se promissoras ao prognóstico do paciente, evidenciando um campo favorável de pesquisa.

Por fim, a magnitude da COVID-19 no cenário mundial, com crescentes número de casos e de óbitos e ausência de consensos que embasam o tratamento do paciente, faz com que estudos que evidenciem terapêuticas que possam ser utilizadas na alta complexidade para combate a COVID-19 sejam primordiais, uma vez que as complicações decorrentes desta doença culminam com os piores desfechos.

\section{Referências}

Araújo, L. P., Sá, N. M., \& Atty, A. T. de M. (2016). Necessidades Atuais de Radioterapia no SUS e Estimativas para o Ano de 2030. Revista Brasileira De Cancerologia, 62(1), 35-42. https://doi.org/10.32635/2176-9745.RBC.2016v62n1.177 
Ashrafian, H., Bogle, R. G., Rosen, S. D., Henein, M., \& Evans, T. W. (2004) Portable echocardiography. BMJ; 328(7435): 300-301. https://doi.org/10.1136/bmj.328.7435.300

Backes, M. T. S., Erdmann, A. L., \& Büscher, A. (2015). The Living, Dynamic and Complex Environment Care in Intensive Care Unit. Revista LatinoAmericana de Enfermagem, 23(3), 411-418. 2015.https://doi.org/10.1590/0104-1169.0568.2570

Balbinot Filho, C. A., \& Borges, C. D. (2020). Efeitos da radiação UV-C em alface e maçã minimamente processadas: uma revisão. Brazilian Journal of Food Technology, 23, e2018321. https://doi.org/10.1590/1981-6723.32118

Brand, C. I., Fontana, R. T., \& Santos, A. V. (2011). A saúde do trabalhador em radiologia: algumas considerações. Texto \& Contexto - Enfermagem, 20(1), 68-75. https://doi.org/10.1590/S0104-07072011000100008

Cheung, J. C., Ho, L. T., Cheng, J. V., Cham, E., \& Lam, K. N. (2020). Staff safety during emergency airway management for COVID-19 in Hong Kong. The Lancet. Respiratory medicine, 8(4), e19. https://doi.org/10.1016/S2213-2600(20)30084-9

Courtemanche, C., Garuccio, J., Le, A., Pinkston, J., \& Yelowitz, A. (2020). Strong Social Distancing Measures In The United States Reduced The COVID-19 Growth Rate. Health affairs (Project Hope), 39(7), 1237-1246. https://doi.org/10.1377/hlthaff.2020.00608

Dhawan, G., Kapoor, R., Dhawan, R., Singh, R., Monga, B., Giordano, J., \& Calabrese, E. J. (2020). Low dose radiation therapy as a potential life saving treatment for COVID-19-induced acute respiratory distress syndrome (ARDS). Radiotherapy and oncology: journal of the European Society for Therapeutic Radiology and Oncology, 147, 212-216. https://doi.org/10.1016/j.radonc.2020.05.002

Franco, L.W. (2018) Radiação UV: Efeitos, Riscos e Benefícios à Saúde Humana - Proposta de Sequência Didática para o Ensino de Física. Dissertação de mestrado, Universidade Estadual Paulista, São Paulo, Brasil.

Galasso, M., Feld, J. J., Watanabe, Y., Pipkin, M., Summers, C., Ali, A., Qaqish, R., Chen, M., Ribeiro, R., Ramadan, K., Pires, L., Bagnato, V. S., Kurachi, C., Cherepanov, V., Moonen, G., Gazzalle, A., Waddell, T. K., Liu, M., Keshavjee, S., Wilson, B. C., \& Cypel, M. (2019). Inactivating hepatitis C virus in donor lungs using light therapies during normothermic ex vivo lung perfusion. Nature communications, 10(1), 481. https://doi.org/10.1038/s41467-018-08261-

Guarner, J. (2020). Three Emerging Coronaviruses in Two Decades. American journal of clinical pathology, 153(4), 420-421. https://doi.org/10.1093/ajcp/aqaa029

Haji, K., Wong, C., Neil, C., Cox, N., Mulligan, A., Wright, L., Vogrin, S., \& Marwick, T. H. (2019). Handheld ultrasound to reduce requests for inappropriate echocardiogram (HURRIE). Echo research and practice, 6(4), 91-96. https://doi.org/10.1530/ERP-19-0016

Instituto de Evaluacion de Tecnologias en Salud e Investigación (IETSI). (2020) Uso de la luz ultravioleta como sustituto a la presión negativa en unidades de cuidados intensivos en el contexto de SARS-CoV-2. Peru: Seguro Social de Salud; Reporte breve n ${ }^{\circ}$ 15. http://docs.bvsalud.org/biblioref/2020/05/1095848/rb15-uv-en-ambientes-hospitalarios-v30mar.pdf.

Jacobs, J. P., Stammers, A. H., St Louis, J., Hayanga, J., Firstenberg, M. S., Mongero, L. B., Tesdahl, E. A., Rajagopal, K., Cheema, F. H., Coley, T., Badhwar, V., Sestokas, A. K., \& Slepian, M. J. (2020). Extracorporeal Membrane Oxygenation in the Treatment of Severe Pulmonary and Cardiac Compromise in Coronavirus Disease 2019: Experience with 32 Patients. ASAIO journal (American Society for Artificial Internal Organs: 1992), 66(7), 722730. https://doi.org/10.1097/MAT.0000000000001185

Kirkby, C., \& Mackenzie, M. (2020). Is low dose radiation therapy a potential treatment for COVID-19 pneumonia?. Radiotherapy and oncology: journal of the European Society for Therapeutic Radiology and Oncology, 147, 221. https://doi.org/10.1016/j.radonc.2020.04.004

Lima, C. M. A. O. (2020) Information about the new coronavirus disease (COVID-19). Radiologia Brasil. 53(2): V-VI. 10.1590/0100-3984.2020.53.2e1

Loforte, A., Dal Checco, E., Gliozzi, G., Benedetto, M., Cavalli, G. G., Mariani, C., Piccone, G., Agulli, M., Pacini, D., \& Baiocchi, M. (2020). Veno-venous Extracorporeal Membrane Oxygenation Support in COVID-19 Respiratory Distress Syndrome: Initial Experience. ASAIO journal (American Society for Artificial Internal Organs: 1992), 66(7), 734-738. https://doi.org/10.1097/MAT.0000000000001198

McMahon, S. R., De Francis, G., Schwartz, S., Duvall, W. L., Arora, B., \& Silverman, D. I. (2020). Tablet-Based Limited Echocardiography to Reduce Sonographer Scan and Decontamination Time during the COVID-19 Pandemic. Journal of the American Society of Echocardiography: official publication of the American Society of Echocardiography, 33(7), 895-899. https://doi.org/10.1016/j.echo.2020.05.005

Ministério da Saúde (BR). Secretaria de Atenção Primária à Saúde. (2020) Protocolo de manejo clínico do coronavírus (COVID-19) na atenção primária à saúde [internet]. 7a versão. Brasília: Ministério da Saúde; 2020. Available from: https://saude.rs.gov.br/upload/arquivos/202004/14140606-4-msprotocolomanejo-aps-ver07abril.pdf

Ministério da Saúde, Secretaria-Executiva (BR). (2009) Área de Economia da Saúde e Desenvolvimento. Avaliação de Tecnologias em Saúde Ferramentas para a Gestão do SUS, Editora do Ministério da Saúde, Brasília, DF, Brasil. https://bvsms.saude.gov.br/bvs/publicacoes/avalia cao_tecnologias_saude_ferramentas_gestao.pdf

Omary, M. B., Eswaraka, J., Kimball, S. D., Moghe, P. V., Panettieri, R. A., Jr, \& Scotto, K. W. (2020). The COVID-19 pandemic and research shutdown: staying safe and productive. The Journal of clinical investigation, 130(6), 2745-2748. https://doi.org/10.1172/JCI138646.

Organização Pan-Americana da Saúde (OPAS). (2020) Folha informativa - COVID-19 (doença causada pelo novo coronavírus). https://www.paho.org/bra/index.php?option=com_content\&view=article\&id=6101:covid19\&Itemid=875

Peters, M. D., Godfrey, C. M., Khalil, H., McInerney, P., Parker, D., \& Soares, C. B. (2015). Guidance for conducting systematic scoping reviews. International journal of evidence-based healthcare, 13(3), 141-146. https://doi.org/10.1097/XEB.0000000000000050 
Research, Society and Development, v. 10, n. 3, e18210313243, 2021

(CC BY 4.0) | ISSN 2525-3409 | DOI: http://dx.doi.org/10.33448/rsd-v10i3.13243

Ramanathan, K., Antognini, D., Combes, A., Paden, M., Zakhary, B., Ogino, M., MacLaren, G., Brodie, D., \& Shekar, K. (2020). Planning and provision of ECMO services for severe ARDS during the COVID-19 pandemic and other outbreaks of emerging infectious diseases. The Lancet. Respiratory medicine, 8(5), 518-526. https://doi.org/10.1016/S2213-2600(20)30121-1

Savino, K., \& Ambrosio, G. (2019). Handheld Ultrasound and Focused Cardiovascular Echography: Use and Information. Medicina (Kaunas, Lithuania), 55(8), 423. https://doi.org/10.3390/medicina55080423

Souza, J.F. (2014) Utilização de Luz Ultravioleta Contínua (Uv-C) e Luz Pulsada Para Conservação De Mangas Cv. Tommy Atkins Minimamente Processadas. Tese de doutorado, Universidade Estadual Paulista, São Paulo, Botucatu, SP, Brasil.

Souza, N., Santos, I., Bushatsky, M., Figueiredo, E., Melo, J., \& Santos, C. (2017). Atuação de enfermeiros em serviços de radioterapia. Revista Enfermagem UERJ, 25, e26130.https://doi.org/10.12957/reuerj.2017.26130

Telford, L. H., Abdullahi, L. H., Ochodo, E. A., Zühlke, L. J., \& Engel, M. E. (2018). Standard echocardiography versus handheld echocardiography for the detection of subclinical rheumatic heart disease: protocol for a systematic review. BMJ open, 8(2), e020140. https://doi.org/10.1136/bmjopen-2017-020140.

Tricco, A. C., Lillie, E., Zarin, W., O'Brien, K. K., Colquhoun, H., Levac, D., Moher, D., Peters, M., Horsley, T., Weeks, L., Hempel, S., Akl, E. A., Chang, C., McGowan, J., Stewart, L., Hartling, L., Aldcroft, A., Wilson, M. G., Garritty, C., Lewin, S., \& Straus, S. E. (2018). PRISMA Extension for Scoping Reviews (PRISMA-ScR): Checklist and Explanation. Annals of internal medicine, 169(7), 467-473. https://doi.org/10.7326/M18-0850.

Ueki, S. Y. M., Geremias, A. L., Moniz, L. L., Latrilha, F. O., Brito, A. C., Giampaglia, C. M. S., Simeão, F. C. S., \& Telles, M. A. S. (2006). Cabine de segurança biológica: efeito da luz ultravioleta nas micobactérias. Revista do Instituto Adolfo Lutz (Impresso), 65(3), 222-224. http://periodicos.ses.sp.bvs.br/scielo.php?script=sci_arttext\&pid=S0073-98552006000300014\&lng=pt\&tlng=pt.

Wong, D. Y., Ranganath, T., \& Kasko, A. M. (2015). Low-Dose, Long-Wave UV Light Does Not Affect Gene Expression of Human Mesenchymal Stem Cells. PloS one, 10(9), e0139307. https://doi.org/10.1371/journal.pone.0139307

World Health Organization (WHOa). (2020) Coronavirus disease (COVID-19): Situation report - 189. https://www.who.int/emergencies/diseases/novelcoronavirus-2019/situation-reports

World Health Organization (WHOb). (2020) Report of the WHO-China Joint Mission on Coronavirus Disease 2019 (COVID-19). https://www.who.int/docs/default-source/coronaviruse/who-china-joint-mission-on-covid-19-final-report.pdf.

World Health Organization (WHOc). (2020) Cleaning and disinfection of environmental surfaces in the context of COVID-19. https://www.who.int/publications/i/item/cleaning-and-disinfection-of-environmental-surfaces-inthe-context-of-covid-19

Yamano, N., Kunisada, M., Kaidzu, S., Sugihara, K., Nishiaki-Sawada, A., Ohashi, H., Yoshioka, A., Igarashi, T., Ohira, A., Tanito, M., \& Nishigori, C. (2020). Long-term Effects of 222-nm ultraviolet radiation C Sterilizing Lamps on Mice Susceptible to Ultraviolet Radiation. Photochemistry and photobiology, 96(4), 853-862. https://doi.org/10.1111/php.13269

Zeng, Y., Cai, Z., Xianyu, Y., Yang, B. X., Song, T., \& Yan, Q. (2020). Prognosis when using extracorporeal membrane oxygenation (ECMO) for critically ill COVID-19 patients in China: a retrospective case series. Critical care (London, England), 24(1), 148. https://doi.org/10.1186/s13054-020-2840-8

Zhang, L., Wang, B., Zhou, J., Kirkpatrick, J., Xie, M., \& Johri, A. M. (2020). Bedside Focused Cardiac Ultrasound in COVID-19 from the Wuhan Epicenter: The Role of Cardiac Point-of-Care Ultrasound, Limited Transthoracic Echocardiography, and Critical Care Echocardiography. Journal of the American Society of Echocardiography: official publication of the American Society of Echocardiography, 33(6), 676-682. https://doi.org/10.1016/j.echo.2020.04.004 